\title{
A Threshold Logic Synthesis Tool for RTD Circuits
}

\author{
María J. Avedillo and José M. Quintana \\ Instituto de Microelectrónica de Sevilla, IMSE-CNM, \\ Universidad de Sevilla, SPAIN.
}

\begin{abstract}
Functional devices and circuits based on Resonant Tunnelling Diodes (RTDs) are receiving much attention since they allow high speed and/or low power operation. RTDs exhibit a negative differential resistance in their current-voltage characteristic which can be exploited to significantly increase the functionality implemented by a single gate in comparison to other technologies. In particular they have proven to efficiently implement threshold gates which are a generalization of conventional boolean gates. Suitable logic synthesis tools are required to handle these complex building blocks in order to translate the advantages of this emergent technology to the circuit and system levels. This paper describes an efficient approach to the automatic design of networks of threshold gates from functional specifications. Results on wide used logic functions and standard benchmark circuits are reported.
\end{abstract}

\section{INTRODUCTION}

Resonant tunnelling diodes (RTDs) are very fast non linear circuit elements which are used in high speed low-power circuits. Switching speeds at room temperature in the order of one picosecond have been reported for these devices. In addition, RTDs exhibit a negative differential resistance (NDR) region in their current-voltage characteristics which can be exploited to significantly increase the functionality implemented by a single gate in comparison to MOS and bipolar technologies, thus reducing circuit complexity and power consumption. Many of them rely on utilizing the latching property of the clocked series connection of a pair of RTDs (MOBILE) arising from their NDR characteristic. In general, MOBILE based logic families combine the basic pair of series-connected RTDs with different three terminal devices to achieve input-output isolation and functionality. Most reported working circuits have been fabricated in III/V while Sibased RTDs is an area of active research.
The MOBILE operating principle [1] is very well suited to implement Threshold Gates (TGs) [2]. RTD-based TGs have been fabricated and have demonstrated high speed and robust operation [4], [5]. The power of the threshold gates lies in the intrinsic complex functions implemented by such gates, which allows for realizations that require less threshold gates than standard boolean logic gates. A number of theoretical results show that polynomial-size, bounded level networks of threshold gates can implement functions that require unbounded level networks of standard logic gates In particular, important functions like multiple-addition, multiplication, division, or sorting can be implemented by polynomialsize threshold circuits of small constant depth [3].

Translating the advantages of this emergent technology to the circuit and system levels could be limited by the lack of automatic synthesis procedures. Many logic algorithm exist targeting conventional logic gates but few have been specifically developed for TGs. The problem was addressed as early as the beginning of the 70 's, but unfortunately it seems that almost nothing has been done since then. LSAT algorithm [6] inspired from techniques used in classical two-level minimization of logic circuits, a multi-level approach [7] and a very recent work [8] are remarkable exceptions.

This paper describes an efficient approach to the automatic design of networks of threshold gates from functional especifications. Set of solutions are described by discrete functions and represented by Multi-valued Decision Diagrams (MDDs) which are a variant of BDDs. The problem of designing a optimum network of TGs is transformed in a satisfiability problem. The rest of the paper is organized as follows. Section II formally defines the concept of threshold logic and establish the formulation of the problem of logic synthesis for TGs on which our CAD tool relies. Section III describes the implementation of the proposed approach. Section IV reports experimental results. Finally, Section V gives some conclusions. 


\section{LOGIC SYNTHESIS BASED ON THRESHOLD GATES}

\subsection{Background}

A threshold gate (TG) is defined as a logic gate with $n$ binary input variables, $x_{i},(i=1, \ldots, n)$, one binary output $y$, and for which there is a set of $(n+1)$ real numbers: threshold $T$ and weights $w_{1}, w_{2}, \ldots, w_{n}$, such that its inputoutput relationship is defined as $y=1$ iff $\sum_{i=1}^{n} w_{i} x_{i} \geq T$ and $y=0$ otherwise [2]. Sum and product are the conventional, rather than the logical, operations. Figure 1 shows the non-standard symbol we use for TGs.

Many CAD problems can be naturally formulated by means of discrete functions by defining a characteristic function $F$ which implicitly represents the set of solutions:

$$
\begin{aligned}
& F\left(\mathrm{c}_{1}, \mathrm{c}_{2}, \ldots, \mathrm{c}_{n}\right)=1 \text { iff }\left(\mathrm{c}_{1}, \mathrm{c}_{2}, \ldots, \mathrm{c}_{n}\right) \text { is a solution } \\
& F\left(\mathrm{c}_{1}, \mathrm{c}_{2}, \ldots, \mathrm{c}_{n}\right)=0 \text { in othercase }
\end{aligned}
$$

where $c_{1}, c_{2}, \ldots, c_{n}$ are multi-valued variables which can take values from a discrete set. Thus, given $F$, the CAD problem is solved by determining whether $F$ is satisfiable, that is, whether it evaluates to 1 for some input combination and in such case, obtaining one or the complete set of such input combinations verifying $F$. It

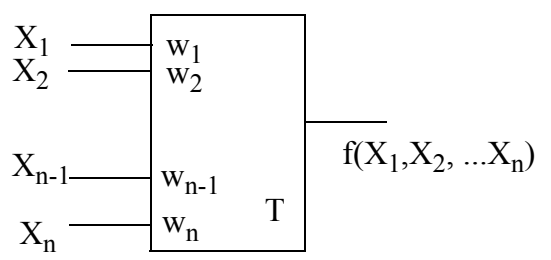

Figure 1.- Threshold gate symbol.

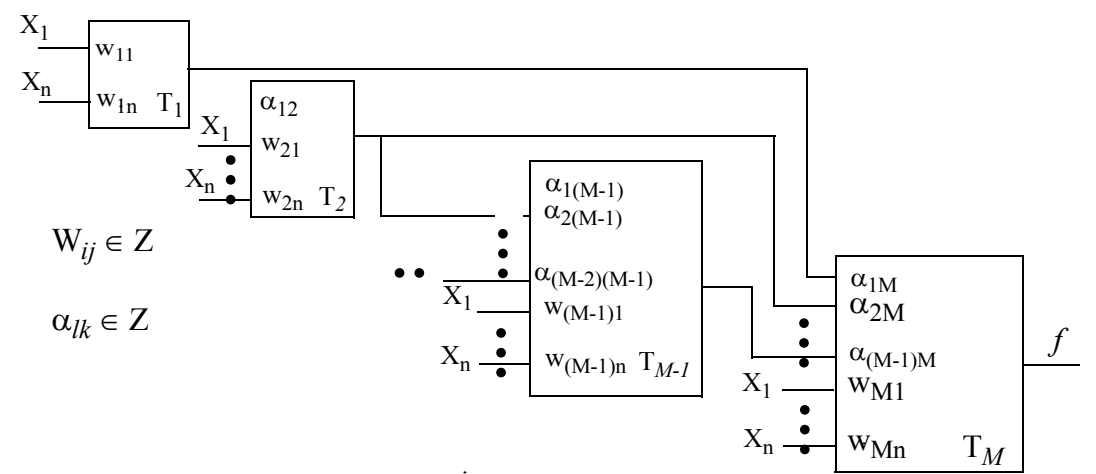

Figure 3.- General multi-layer feed forward network of threshold gates is very important to efficiently realize this using a suitable representation for $F$. We have resorted to MDDs that we briefly introduced.

It is well known that binary boolean functions, $f: \mathrm{B}^{n} \rightarrow \mathrm{B}$, can be represented by ordered Binary Decision Diagrams (BDD) [9]. A BDD is a directed acyclic graph where a Shannon decomposition is carried out in each node

BDDs can be extended to Multivalued Decision Diagrams (MDDs) [10], [11] representing multivalued functions, $f:\{0,1, \ldots$, $k-1\}^{n} \rightarrow \mathrm{B}$. Each internal node has as many outgoing edges as different values can take the variables. Figure 2 shows an MDD of the characteristic function of the relation $x>y$, with $x$ and $y \in\{0$, $1,2\}$.

\subsection{Network synthesis}

Figure 3 depicts a multi-layer feed forward network of threshold logic which is able to implement any $n$-input function $f\left(\mathrm{x}_{1}, \mathrm{x}_{2}\right.$, $\left.\ldots, x_{n}\right)[2]$.

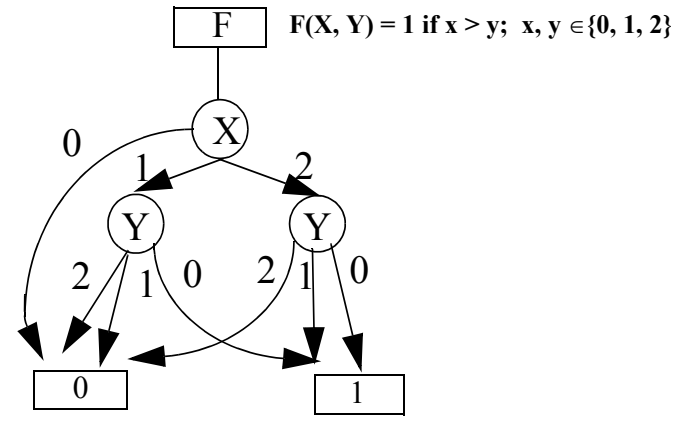

Figure 2.- MDD of $\mathbf{x}>\mathbf{y}$. 
1) A multi-valued integer variable is associated to each of the weights and threshold in the network.

2) For each input combination $\left(x_{1}, x_{2}, \ldots, x_{n}\right)$ for which $f\left(x_{1}, x_{2}, \ldots, x_{n}\right)=1$ the following function with binary output and multi-valued input is built up ${ }^{1}$ :

$$
\begin{gathered}
\left(\sum_{i=1}^{n} W_{M i} \cdot x_{i}+\alpha_{1 M}+\ldots+\alpha_{(M-1) M} \geq T_{M}\right)\left(\sum_{i=1}^{n} W_{(M-1) i} \cdot x_{i}+\alpha_{1(M-1)}+\ldots+\alpha_{(M-2)(M-1)} \geq T_{M-1}\right) \ldots\left(\sum_{i=1}^{n} W_{1 i} \cdot x_{i} \geq T_{1}\right) \| \\
\left(\sum_{i=1}^{n} W_{M i} \cdot x_{i}+\alpha_{2 M} \ldots+\alpha_{(M-1) M} \geq T_{M}\right)\left(\sum_{i=1}^{n} W_{(M-1) i} \cdot x_{i}+\ldots+\alpha_{(M-2)(M-1)} \geq T_{M-1}\right) \ldots\left(\sum_{i=1}^{n} W_{1 i} \cdot x_{i}<T_{1}\right) \| \\
\ldots \\
\left(\sum_{i=1}^{n} W_{M i} \cdot x_{i} \geq T_{M}\right)\left(\sum_{i=1}^{n} W_{(M-1) i} \cdot x_{i}<T_{M-1}\right) \ldots\left(\sum_{i=1}^{n} W_{1 i} \cdot x_{i}<T_{1}\right)
\end{gathered}
$$

3) For each input combination $\left(x_{1}, x_{2}, \ldots, x_{n}\right)$ for which $f\left(x_{1}, x_{2}, \ldots, x_{n}\right)=0$ the following function with binary output and multi-valued input is built up ${ }^{1}$

$$
\begin{gathered}
\left(\sum_{i=1}^{n} W_{M i} \cdot x_{i}+\alpha_{1 M}+\ldots+\alpha_{(M-1) M}<T_{M}\right)\left(\sum_{i=1}^{n} W_{(M-1) i} \cdot x_{i}+\alpha_{1(M-1)}+\ldots+\alpha_{(M-2)(M-1)} \geq T_{M-1}\right) \ldots\left(\sum_{i=1}^{n} W_{1 i} \cdot x_{i} \geq T_{1}\right) \| \\
\left(\sum_{i=1}^{n} W_{M i} \cdot x_{i}+\alpha_{2 M}+\ldots \alpha_{(M-1) M}<T_{M}\right)\left(\sum_{i=1}^{n} W_{(M-1) i} \cdot x_{i}+\ldots+\alpha_{(M-2)(M-1)} \geq T_{M-1}\right) \ldots\left(\sum_{i=1}^{n} W_{1 i} \cdot x_{i}<T_{1}\right) \| \\
\left(\sum_{i=1}^{n} W_{M i} \cdot x_{i}<T_{M}\right)\left(\sum_{i=1}^{n} W_{(M-1) i} \cdot x_{i}<T_{M-1}\right) \ldots\left(\sum_{i=1}^{n} W_{1 i} \cdot x_{i}<T_{1}\right)
\end{gathered}
$$

4) Function $F_{\text {redM }}$ is built up from the conjuction of the expresions obtained in previous steps of this procedure.

1.1Each parenthesis represents the characteristic function of the corresponding relation.

Figure 4.- Procedure to synthesize a network with $M$ threshold gates

The problem of logic synthesis using threshold gates can be formulated as:

Given $f\left(x_{1}, x_{2}, \ldots, x_{n}\right)$ determine the minimum number of threshold elements, $M$, required to implement $f$, as well as the set of weights and threshold for each of the gates

From the description of a function, $f\left(\mathrm{x}_{1}, \mathrm{x}_{2}, \ldots, \mathrm{x}_{n}\right)$, the procedure shown in Figure 4 builds up a multi-valued function, $F_{\text {redM }}$, such that if this function is satisfiable then can be implemented by a network with $M$ gates and the structure in Figure 3. Each assignment of input variables satisfying $F_{\text {redM }}$ completely defines a network implementing $f$. That is, specifies weights and threshold for each gate. The problem of optimum synthesis of threshold gates networks can be solved in an incremental way by solving a sequence of problems, repeating the described procedure for increasing values of $M$.
Given the NP nature of this problem, the search of exact solutions is only practical for a low number of inputs. Circuit partition techniques can be used and the synthesis procedure just described can be applied to each partition. This is the strategy we have taken in the new tool.

\section{Description of Proposed ApProach}

Figure 5 depicts the block diagram of the tool. It takes as input the functional description of the circuit to be synthesized in ESPRESSO or BLIF format, the ranges for the weights associated to each of the variables as well as the ranges for each of the thresholds values. The output is a netlist of threshold gates. There are two main modules in the tool. The first is the partitioning module. The second module is the synthesis module. It starts with an analysis step which carries out operations such as classification of variables and identification of symmetries with the aim of simpli- 
fying the network synthesis. All these task are realized on the BDD representing the function $f$. Then, it builds the MDDs representing the functions $\mathrm{F}_{\text {red } M}$ described in previous Section. If $F_{\text {red }} M$ can be satisfied, an input assignment verifying it is produced.

The building and storing of the MDD representing can be computationally expensive for functions with a relatively low number of input variables. Because of this, an strategy which transform the problem in a collection of subproblems which can be efficiently solved has been applied (search strategy 1) in our implementation. Each of these subproblem is obtained by fixing a reduced number of he variables.

In addition, it is possible to speed up the search process without carrying out the AND operation of the MDDs associated to the equations corresponding to each input combination. A recursive procedure to obtain an assignment simultaneously satisfying all the sub-functions (search strategy 2) has been developed.

The new tool can be used to exactly solved the problem of deriving the minimum threshold-gate network implementing a given function if no partition is applied. Also, a particular case of using the tool corresponds to specify the range for the weights and for the threshold. In this way, networks of NOR gates are synthesized.

\section{EXPERIMENTAL RESULTS}

A prototype of the tool, called LTHRES, has been implemented using the MDDs' and BDDs' packages from SIS. It also incorporates an standard partition algorithm from SIS [12]. An specific one aiming at optimize for threshold network implementation the partition obtained is currently under development.

Table I shows the results obtained for some logic functions widely used in digital design. The tool has been applied to obtain minimum networks of TGs implementing them. There have been included columns for the number of inputs $(I)$, the number of outputs $(O)$, the minimum number of TGs in a network implementing the functions and the time (seconds in a SparcStation 10) LTHRES consumes in obtaining the complete set of solutions and a single solution with different search strategies. The application of search strategies 1 and 2 is advantageous for the examples with more inputs and outputs.

Table II compares threshold based realizations with implementations using a conventional cell library. Conventional multi-level implementations have been generated within SIS. Optimization has been performed applying script.rugged. Technological mapping has been done by an algorithm targeting area minimization (map - $m 0$ de SIS). The used library comprises 13 gates: in, nand2, nand3, nand4, nor2, nor 3, nor4, and2, or2, aoi21, aoi22, oai21 y oai22. The resulting implementations have been compared in terms of number of gates and logic levels. In order to estimate the complexity of implementing these circuits with less levels, the number of product terms ( $t p)$ in a two-level implementation obtained with ESPRESSO is also included.

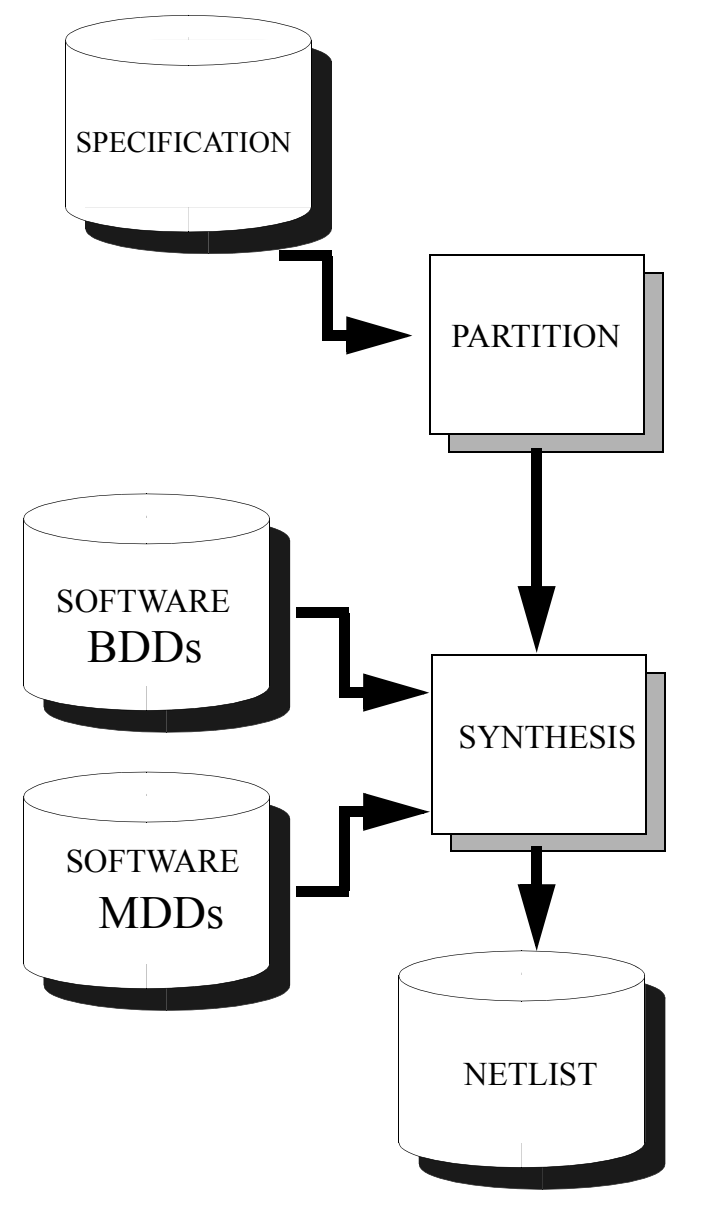

Figura 5.- Block diagram of new tool

Finally, Table III compares threshold and conventional realizations for a set of standard benchmark circuits. Both implementations have been obtained applying identical combinational optimization. In the partitioning carried out to derive threshold network the number of inputs per partition has been limited to 5 . Even without an specific partitioning algorithm good results are obtained in spite of the fact that a specific partition algortihm is not being applied.

Comparisons to other existing tools have not carried out. It has no sense in the case of LSAT which is aimed at two-level implementations. Concerning the multi-level approach from [8], it has not been possible as the tool is not available. Direct comparison of data reported is not significant because several reasons. First, because there are not enough details on combinational optimization to be able to reproduce it. Second, reported results constraint fan-in of the threshold gates to three. 
Table I: Synthesis of minimum networks with different options of LTHRES

\begin{tabular}{|c|c|c|c|c|c|c|c|c|}
\hline & \# I & \# O & $\begin{array}{c}\# \\
\text { gates }\end{array}$ & $\underset{\text { time }(\mathrm{s})}{\mathrm{a}}$ & $\stackrel{b}{\text { time }(s)}$ & $\underset{\text { time }(s)}{c}$ & $\underset{\text { time }(\mathrm{s})}{\mathrm{d}}$ & $\underset{\text { time (s) }}{\mathrm{e}}$ \\
\hline par3 & 3 & 1 & 2 & $1^{\prime} 9$ & $2 ’ 3$ & 3 & $1 ' 3$ & $2 ’ 3$ \\
\hline par4 & 4 & 1 & 3 & $263 ’ 2$ & 408 & 414 & 93 & 202 \\
\hline par5 & 5 & 1 & 3 & 669 & 1160 & 1186 & 373 & 1130 \\
\hline d.ff & 4 & 1 & 2 & $6^{\prime} 6$ & $10^{\prime} 4$ & $10^{\prime} 4$ & $3 ' 2$ & $6^{\prime} 9$ \\
\hline d2.ff & 4 & 1 & 2 & 7'1 & $10 ’ 2$ & $10^{\prime} 1$ & $2 ' 8$ & $5^{\prime} 6$ \\
\hline $2 \mathrm{de} 5$ & 5 & 1 & 2 & $40^{\prime} 6$ & 67 & 67 & 30 & 78 \\
\hline add & 4 & 3 & 7 & 581 & 573 & 219 & 95 & 101 \\
\hline addc & 5 & 3 & 7 & $>1200$ & 481 & 479 & 309 & 182 \\
\hline
\end{tabular}

a.- Obtaining the complete set of solutions

b.- Obtaining the complete set of solutions with search strategy 1.

c.- . Obtaining a single solution with search strategy 1.

d.- Obtaining a single solution with search strategy 2.

e.- Obtaining a single solution with search strategy 1 and 2.

time seconds in Sparc10

Table II:Comaprison of threshold and conventional realization

\begin{tabular}{|c|c|c|c|c|c|c|c|}
\hline & \# I & \# O & $\begin{array}{c}\text { Thres. } \\
\text { \# gates }\end{array}$ & \# levels & $\begin{array}{c}\text { Conv. } \\
\text { \# gates }\end{array}$ & \# levels & $\begin{array}{c}\text { Two-Levels\# } \\
\text { tp }\end{array}$ \\
\hline \hline par3 & 3 & 1 & 2 & 2 & 4 & 4 & 4 \\
\hline par4 & 4 & 1 & 3 & 3 & 7 & 6 & 8 \\
\hline par5 & 5 & 1 & 3 & 3 & 8 & 8 & 16 \\
\hline d.ff & 4 & 1 & 2 & 2 & 3 & 3 & 2 \\
\hline d2.ff & 4 & 1 & 2 & 2 & 3 & 3 & 2 \\
\hline 2 de5 & 5 & 1 & 2 & 2 & 15 & 5 & 11 \\
\hline add & 4 & 3 & 7 & 2 & 13 & 7 & 23 \\
\hline addc & 5 & 3 & 7 & 2 & 16 & 8 & 2 \\
\hline
\end{tabular}




\section{CONCLUSIONS}

A novel logic synthesis tool for the synthesis of general boolean functions using threshold gates has been described. It is based on the representation of the discrete characteristic function representing the set of solutions to the problem of implementing a given function with a multi-layer feed-forward network of threshold functions with $M$ gates. Since this exact solution is not practical when the number of inputs increases, the circuit is partitioned in clusters with are then exactly solved. Preliminary results using an standard partitioning algorithm, that is, non specifically optimized for the threshold network implementation it is targeted, are very promising.

Addressing the problem of synthesis with threshold gates is extremely important for the success of emergent technologies like RTDs which application could be limited by the lack of CAD tools.

\section{REFERENCES}

[1] K.J. Chen, K. Maezawa and M. Yamamoto: "InP-Based High Performance Monostable-Bistable Transition Logic Elements (MOBILEs) Using Integrated MultipleInput Resonant-Tunneling Devices," IEEE Electron Device Letters, Vol. 17, no. 3, pp. 127-129, March 1996.

[2] S. Muroga, "Threshold Logic \& its Applications", Wiley-Interscience 1971.

[3] K.Y. Siu, W.P. Toychowdhury y T. Kailath, "Deph-Size Tadeoffs for Neural Computations", IEEE Trans. on Computers, Vol. 40, no. 12, pp. 1402-1411. Dec. 1991.

[4] C. Pacha et al.: "Threshold Logic Circuit Design of Parallel Adders Using Resonant Tunnelling Devices," IEEE Trans. on VLSI Systems, Vol. 8, no. 5, pp. 558-572, Oct. 2000.

[5] M.J. Avedillo, J.M. Quintana, H. Pettenghi, P.M. Kelly, and C.J. Thompson, "Multi-threshold Threshold Logic Circuit Design Using Resonant Tunneling Devices," Electronics Letters, Vol. 39, pp. 1502-1504, 2003.

[6] A.L. Oliveira y A. Sangiovanni, "LSAT-An Algorithm for the Synthesis of Two-Level Threshold Gate Networks", Proc. of Int. Conf. of Computer-Aided Design, pp. 130-133, 1991.

[7] A. Gonzalez, M.J. Avedillo, J.M. Quintana and A. Rueda: "Una Herramienta de Síntesis Lógica para Realizaciones basadas en Puertas Umbral", Proceedings of 10th Congreso de Diseño de Circuitos Integrados, pp. 230-235, Zaragoza, Spain 1995.

[8] R. Zhang, P. Gupta, L. Zhong, and N.K. Jha: "Synthesis and optimization of Threshold Logic Networks with applications to nanotechnologies", Proceedings DATE 2004.
[9] R. E. Bryant, "Graph Based Algorithms for Boolean Function Manipulation”, IEEE Trans. on Computers, C35(8):677-691, August 1986.

[10] T. Kam, "Multi-valued Decision Diagrams" Master Thesis, Universidad de Berkeley, 1990.

[11] A. Srinivasan, T. Kam, S. Malik y R. Brayton, "Algorithms for Discrete Function Manipulation", Proc. of Int. Conf. of Computer-Aided Design, pp. 92 -95, Noviembre 1990.

[12]E.M. Sentovich et al., "SIS: A System for Sequential Circuit Synthesis", Memo UCB/ERL M92/41, Univ. California, Berkeley, May, 1992.

Table III:Results of LTHRES with standard partition algorithm

\begin{tabular}{|c|c|c|c|c|}
\hline & \# I & $\# \mathrm{O}$ & $\begin{array}{l}\text { Conv. } \\
\text { \# gates }\end{array}$ & $\begin{array}{l}\text { Thres. } \\
\text { \# gates }\end{array}$ \\
\hline 9symml & 9 & 1 & 107 & 17 \\
\hline alu2 & 10 & 6 & 218 & 174 \\
\hline b9 & 41 & 21 & 82 & 64 \\
\hline cm138a & 6 & 8 & 19 & 10 \\
\hline cm42a & 4 & 10 & 20 & 11 \\
\hline cm82a & 5 & 3 & 14 & 6 \\
\hline decod & 5 & 16 & 32 & 16 \\
\hline cm85a & 11 & 3 & 32 & 12 \\
\hline cm151a & 12 & 2 & 12 & 13 \\
\hline cu & 14 & 11 & 35 & 24 \\
\hline cmb & 16 & 4 & 32 & 18 \\
\hline cm163a & 16 & 5 & 22 & 21 \\
\hline cm162a & 14 & 5 & 27 & 21 \\
\hline ce & 21 & 20 & 42 & 26 \\
\hline cm150a & 21 & 1 & 40 & 24 \\
\hline
\end{tabular}

